\title{
Two Properties of a Gene-for-Gene Coevolution System under Human Perturbations
}

\author{
P. Sun and X. B. Yang
}

Department of Plant Pathology, Iowa State University, Ames 50011.

Accepted for publication 12 April 1999.

\section{ABSTRACT}

Sun, P., and Yang, X. B. 1999. Two properties of a gene-for-gene coevolution system under human perturbations. Phytopathology 89:811-816.

Recently, the gene-for-gene host-parasite coevolution model of Leonard was extended by incorporating two kinds of perturbations. The first kind was the natural perturbations that include those caused by pathogen migration between the two subpopulations of the host, forward and backward mutations in the host or pathogen populations, and some others. The second kind was human perturbations, such as constantly increasing the percentage of the resistant genotype within the host population each season. In this study, we quantitatively compared the two kinds of perturbations and extended the constantly changing human perturbation to include non- constant perturbations that are more likely to occur in the real world. Two properties of the modified Leonard model were revealed from this study. First, when both human perturbations and natural perturbations are involved, the effects of natural perturbations are very small compared with those of human perturbations. This finding ensures that, in the study of human perturbations, we can simplify the study by ignoring the effects of natural perturbations. Second, through the simulation of nonconstant perturbations, which assumes that the proportion of the resistant genotype of the host population increases over time, we found that the model reproduces the "boom and bust" epidemic cycles that are often found in agroecosystems.

Additional keywords: host-parasite interaction, population dynamics.
Population dynamics of a natural host-parasite system have been described using a nonlinear model by Leonard $(9,10)$. In reality, a host-parasite system is always perturbed by different factors. Recent studies have demonstrated that perturbations could significantly change the dynamics of the host-parasite system described by Leonard's model $(8,11,17)$. In general, two kinds of perturbations are considered. The first is natural, which includes those caused by pathogen migration between two host subpopulations, forward and backward mutations in the host and pathogen populations, and others $(8,11)$. The second is human perturbations, such as the theoretical addition of a constant percentage of resistant genotypes to the host population each generation (17).

Computer simulations show that under some sets of parameter values the frequencies of the virulent genotype in the parasite population, $n_{i}$, and the resistant genotype, $p_{i}$, in the host population in Leonard's model would converge to the internal stable equilibrium point (EP) at a very slow rate. It is almost impossible for the frequencies $\left(n_{i}, p_{i}\right)$ to reach the internal $\mathrm{EP}$ within a limited number of generations $(10,17)$. The rate of convergence, however, increases drastically with either of the two kinds of perturbations, and the internal EP can be more readily reached under the constant perturbations $(8,11,17)$.

Disease dynamics in a nonnatural host-parasite system are always perturbed both by natural and human perturbations. Modeling the dynamics of a host-parasite interaction in systems that are affected by perturbations are extensions of models from natural systems. These systems extended by including perturbation factors are closer to the actual host-parasite systems plant pathologists have worked on. In this paper, we further studied the properties of Leonard's model related to perturbations with two aims. One was to study the interactions between the two types of perturbations. Although human perturbations have been considered major forces in patho-

Corresponding author: X. B. Yang; E-mail address: xbyang@iastate.edu

Publication no. P-1999-0520-01R

(C) 1999 The American Phytopathological Society gen evolution in agroecosystems, studies are not available to quantitatively separate the effects of the two perturbations experimentally or theoretically. Such studies may be helpful for the prediction of the long-term dynamics of host-parasite interactions in systems perturbed by human activities. The other aim was to further study the effects of human perturbations on host-parasite systems. In actuality, people do not always use resistant plants to replace susceptible plants at a constant rate in each generation. The percentage of the host plants with genetic resistance added to the host population may be variable.

\section{THEORY}

Leonard's model was simulated by assuming that, in each generation, the percentage of the resistant gene added to the host population is an increasing function. The studies on the host-parasite system from the above two aspects may provide insights of how human perturbations affect the evolution of a plant pathogen in an agroecosystem, which is useful to pathosystem modeling or further analysis on the host-parasite interaction described by Leonard's model.

The original model by Leonard is as follows.

$$
\begin{gathered}
n_{i+1}=g\left(p_{i}, n_{i}\right)=\frac{n_{i}\left[1-k+\left(1-q_{i}^{2}\right) a\right]}{1-\left(1-q_{i}^{2}\right) t+n_{i}\left[\left(1-q_{i}^{2}\right)(a+t)-k\right]} \\
p_{i+1}=f\left(p_{i}, n_{i+1}\right)=\frac{p_{i}\left[1-c-s(1-t)+n_{i+1} s(k-a-t)\right]}{1-s+n_{i+1} k s+\left(1-q_{i}^{2}\right)\left[t s-c-n_{i+1} s(a+t)\right]}
\end{gathered}
$$

in which $n_{i+1}$ is the frequency of the genotype for virulence in the parasite population at the end of the $(i+1)$ th generation and can be denoted by a function $g\left(p_{i}, n_{i}\right)$ (the frequency of the genotype for avirulence is $\left.m_{i+1}\left[m_{i+1}+n_{i+1}=1\right]\right) ; p_{i+1}$ is the frequency of genotype for resistance in the host population at the end of the $(i+1)$ th 
generation and can be denoted by a function $f\left(p_{i}, n_{i+1}\right)$ (the frequency of the genotype for susceptibility is $\left.q_{i+1}\left[p_{i+1}+q_{i+1}=1\right]\right) ; a$ determines the type of selection in the parasite; $c$ represents the fitness cost of resistance in the host population; $k$ is the cost of virulence; $t$ represents the effectiveness of the resistance; and $s$ reflects disease severity. More detailed descriptions of the parameters are described by Leonard $(9,10)$.

Comparison of natural and human perturbations on the stability of host-parasite interaction. Natural perturbations have been shown to affect the rate of a system in reaching a stable status $(8,11)$. Among the natural perturbations, mutation in the host and pathogen populations is regarded by Kirby and Burdon (8) as the most important to the host-parasite coevolution process in a natural system. The importance of mutational effects has been addressed by other researchers as well $(4,5)$. Below, mutation is used as an example for the study of natural perturbations.

The original Leonard model describes a natural host-parasite system. At the end of the $(i+1)$ th generation, frequencies of the resistant $\left(p_{i+1}\right)$ and the virulent $\left(n_{i+1}\right)$ genotype can be calculated by equation set 1 . If the forward and backward mutations in the host and pathogen populations are considered, the frequencies at the $(i+1)$ th generation are no longer $n_{i+1}=g\left(p_{i}, n_{i}\right)$ and $p_{i+1}=f\left(p_{i}, n_{i+1}\right)$, but rather $n_{i+1}=g\left(p_{i}, n_{i}\right)+$ mutation effect and $p_{i+1}=f\left(p_{i}, n_{i+1}\right)+$ mutation effect.

With the mutation rate, $\mu$, the effect of mutation in each direction in both populations is computed by Kirby and Burdon as follows: $n^{\prime}=n+\mu(1-2 n)$ and $q^{\prime}=q+\mu(1-2 q)(8) ; q$ is the allelic frequency of susceptibility in the host population and $p+q=1$. In our calculations, we use the form $p^{\prime}=p+\mu(1-2 p)$, which is equivalent to $q^{\prime}=q+\mu(1-2 q)$, because it is more convenient to use symbols to be consistent with the original Leonard model, in which the dynamic behaviors of the frequencies $p_{i}$ and $n_{i}$ are investigated. If mutation is considered at the $(i+1)$ th generation, the frequency of virulence in the parasite population is no longer $g\left(p_{i}, n_{i}\right)$, but rather $g\left(p_{i}, n_{i}\right)+\mu\left[1-2 g\left(p_{i}, n_{i}\right)\right]$, and the frequency of resistance in the host population is no longer $f\left(p_{i}, n_{i+1}\right)$, but rather $f\left(p_{i}, n_{i+1}\right)+$ $\mu\left[1-2 f\left(p_{i}, n_{i+1}\right)\right]$.

$$
\begin{gathered}
n_{i+1}=g\left(p_{i}, n_{i}\right)+\mu\left[1-2 g\left(p_{i}, n_{i}\right)\right] \\
p_{i+1}=f\left(p_{i}, n_{i+1}\right)+\mu\left[1-2 f\left(p_{i}, n_{i+1}\right)\right]
\end{gathered}
$$

in which $g\left(p_{i}, n_{i}\right)$ and $f\left(p_{i}, n_{i+1}\right)$ are defined in equation set 1 . In equation set 2 , if $g\left(p_{i}, n_{i}\right)<0.5$, then $\mu\left[1-2 g\left(p_{i}, n_{i}\right)\right]>0$; the effect of mutation equals a positive increment of the virulent genotype in the parasite population. If $g\left(p_{i}, n_{i}\right)>0.5$, then $\mu\left[1-2 g\left(p_{i}\right.\right.$, $\left.\left.n_{i}\right)\right]<0$; the effect of mutation equals a decrement of the virulent genotype in the parasite population. If $f\left(p_{i}, n_{i}\right)<0.5$, then $\mu[1-$ $\left.2 f\left(p_{i}, n_{i}\right)\right]>0$; the effect of mutation equals a positive increment of the resistant genotype in the host population. If $g\left(p_{i}, n_{i}\right)>0.5$, then $\mu\left[1-2 g\left(p_{i}, n_{i}\right)\right]<0$; the effect of mutation equals a decrement of the resistant genotype in the host population.

In the case of human farming perturbations, if there is a constant increment in the percentage of the resistant gene added to the host population each generation, Leonard's model can be modified as follows.

$$
\begin{gathered}
n_{i+1}=g\left(p_{i}, n_{i}\right) \\
p_{i+1}=f\left(p_{i}, n_{i+1}\right)+\lambda
\end{gathered}
$$

in which the parameter $\lambda$ is a positive constant smaller than 1 , satisfying $p_{i+1} \leq 1 . n_{i+1}=g\left(p_{i}, n_{i}\right)$ and $p_{i+1}=f\left(p_{i}, n_{i+1}\right)+\lambda$ can also be considered the initial genotype frequencies at the beginning of the $(i+2)$ th generation (17).
A more detailed description of the perturbed system should be written as

$$
\begin{gathered}
n^{*}{ }_{i+1}=n_{i+1}=g\left(p_{i}, n_{i}\right) \\
p^{*}{ }_{i+1}=f\left(p_{i}, n_{i+1}\right) \\
p_{i+1}=f\left(p_{i}, n_{i+1}\right)+\lambda=p^{*}{ }_{i+1}+\lambda
\end{gathered}
$$

in which $\left(p^{*}{ }_{i+1}, n^{*}{ }_{i+1}\right)$ are the frequencies at the end of the $(i+1)$ th generation and $\left(p_{i+1}, n_{i+1}\right)$ are the initial frequencies of the $(i+2)$ th generation. In the host-parasite system described by equation sets 1 and 2, $n_{i+1}$ and $p_{i+1}$ can represent either the initial frequencies of the $(i+2)$ th generation or the frequencies at the end of the $(i+1)$ th generation. This is an important difference between equation set 2 and equation set 3 or 4 . In equation set $4, p_{i+1}>p^{*}{ }_{i+1}$, because the increment of resistant genotype $\lambda$ is added to the host population at the start of a generation. Combining the two kinds of perturbation, we obtained a new form of Leonard's model as follows.

$$
\begin{gathered}
n^{*}{ }_{i+1}=n_{i+1}=g\left(p_{i}, n_{i}\right)+\mu\left[1-2 g\left(p_{i}, n_{i}\right)\right] \\
p^{*}{ }_{i+1}=f\left(p_{i}, n_{i+1}\right)+\mu\left[1-2 f\left(p_{i}, n_{i+1}\right)\right] \\
p_{i+1}=p^{*}{ }_{i+1}+\lambda
\end{gathered}
$$

Effects of overuse of resistant genotypes. Our previous results (17) and above simulations show that the constant perturbation, consisting of the continued increase of resistant genotypes in the host population, can increase the rate that frequencies of $\left(n_{i}, p_{i}\right)$ reach a stable internal EP. History tells us, however, that when a resistant variety is overused in a pathosystem the system does not necessarily have a stable equilibrium, but rather a "boom and bust" cycle of major epidemics $(14,15)$ due to the production of new pathogenic races that have greater fitness to the resistant genotype. It is necessary to investigate the human perturbations, under which the host-parasite systems do not converge to the stable EP. Just as the studies of the conditions that will lead these systems into convergence, the studies of the conditions leading the systems away from the internal EP will provide clues to managing the coevolutionary process of host-parasite interactions in the real world. In host-parasite systems perturbed by agricultural activities, the "boom and bust" cycles of epidemics caused by the overuse of the pure line resistant genotypes are an important phenomena. Extension of Leonard's model with the consideration of human perturbations may be useful to analyze the phenomena.

Suppose a host-parasite system has two host genotypes, the resistant (R) and the susceptible (S), and one pathogen genotype, the avirulent pathogen genotype, which only attacks the susceptible genotype. With typical human perturbations, the percentage of resistant genotype would increase over time because the pathogen is avirulent to the resistant genotype. The increase in the proportion of resistant genotype in the host population is the "boom" part of the cycle. With the increase of the resistant genotype in the host population, a mutant genotype in the parasite population that could attack both the resistant and the susceptible genotypes would appear. The percentage of the virulent genotype in the parasite population will increase drastically with the increase of the resistant genotype. When the percentage of the virulent genotype reaches a certain level, such as above 0.5 , the host population essentially loses its resistance. This is the "busting" of the resistant genotype. Another supposition on the pathogen population is that the virulent genotype already exists in the host population at a very low proportion. With the increase in the resistant genotype of the host population, the percentage of the virulent pathogen is increased under the selection pressure. 
In the real world, a "boom and bust" cycle always culminates in high frequencies of $\left(n_{i}, p_{i}\right)$ and high disease severity in the fields. When a resistant genotype is no longer resistant to the parasite population, a cycle of the "boom and bust" completes, leading to the selection of new resistant genotypes, which may lead to a new cycle of "boom and bust" epidemics. Though it is difficult to predict when mutation of a virulent parasite genotype will occur, extension of Leonard's model could describe the process of one cycle of the "boom and bust" epidemics when the mutation of the virulent parasite genotype has occurred. The key factor in the "boom and bust" epidemic cycles is the overpopulated resistant genotype in the host-parasite system, which can be simulated by the modified Leonard model (equation set 5).

As previously discussed, effects of natural perturbations are rather small when a sufficiently large human perturbation is considered (e.g., larger than 0.001). In actuality, when a resistant genotype is bred, the increment in frequency of the resistant genotype in the host population larger than 0.01 in each season is not an overestimation $(14,15)$. Thus, the mutation terms in equation 5 can be neglected. In practice, the percentage of the resistant genotype added to the host population each season is not constant, but variable, dependent on production needs. We assume the percentage of the resistant genotype $\left(\lambda_{i}\right)$ added to the host population in generation $i$ is a variable, in which $0<\lambda_{i}<1$ and satisfies $p_{i} \leq 1$. The fol- lowing equation set was modified from equation set 5 based on the above analysis.

$$
\begin{gathered}
n^{*}{ }_{i+1}=n_{i+1}=g\left(p_{i}, n_{i}\right) \\
p^{*}{ }_{i+1}=f\left(p_{i}, n_{i+1}\right) \\
p_{i+1}=f\left(p_{i}, n_{i+1}\right)+\lambda_{i+1}
\end{gathered}
$$

To describe the overuse of the resistant genotype, we suppose that $\lambda_{i}$ is increased each generation. As before, the final frequencies of resistant genotype in a host population at the end of the $(i+1)$ th generation is $\left(n^{*}{ }_{i+1}, p^{*}{ }_{i+1}\right)$ and the initial frequencies of the $(i+2)$ th generation is $\left(n_{i+1}, p_{i+1}\right)$.

\section{RESULTS}

Comparison of natural and human perturbations. In equation set 5 , because $-1 \leq 1-2 g\left(p_{i}, n_{i}\right) \leq 1$, we know $-\mu \leq \mu[1-$ $\left.2 g\left(p_{i}, n_{i}\right)\right] \leq \mu$. Since the mutation rate, $\mu$, is between $10^{-4}$ to $10^{-10}$ (8), the mutation effect on the dynamics of the system is smaller than the perturbation from human agricultural activities if $\lambda$ is sufficiently large. For example, if $\lambda>10^{-3}, \lambda$ would be at least 10 times
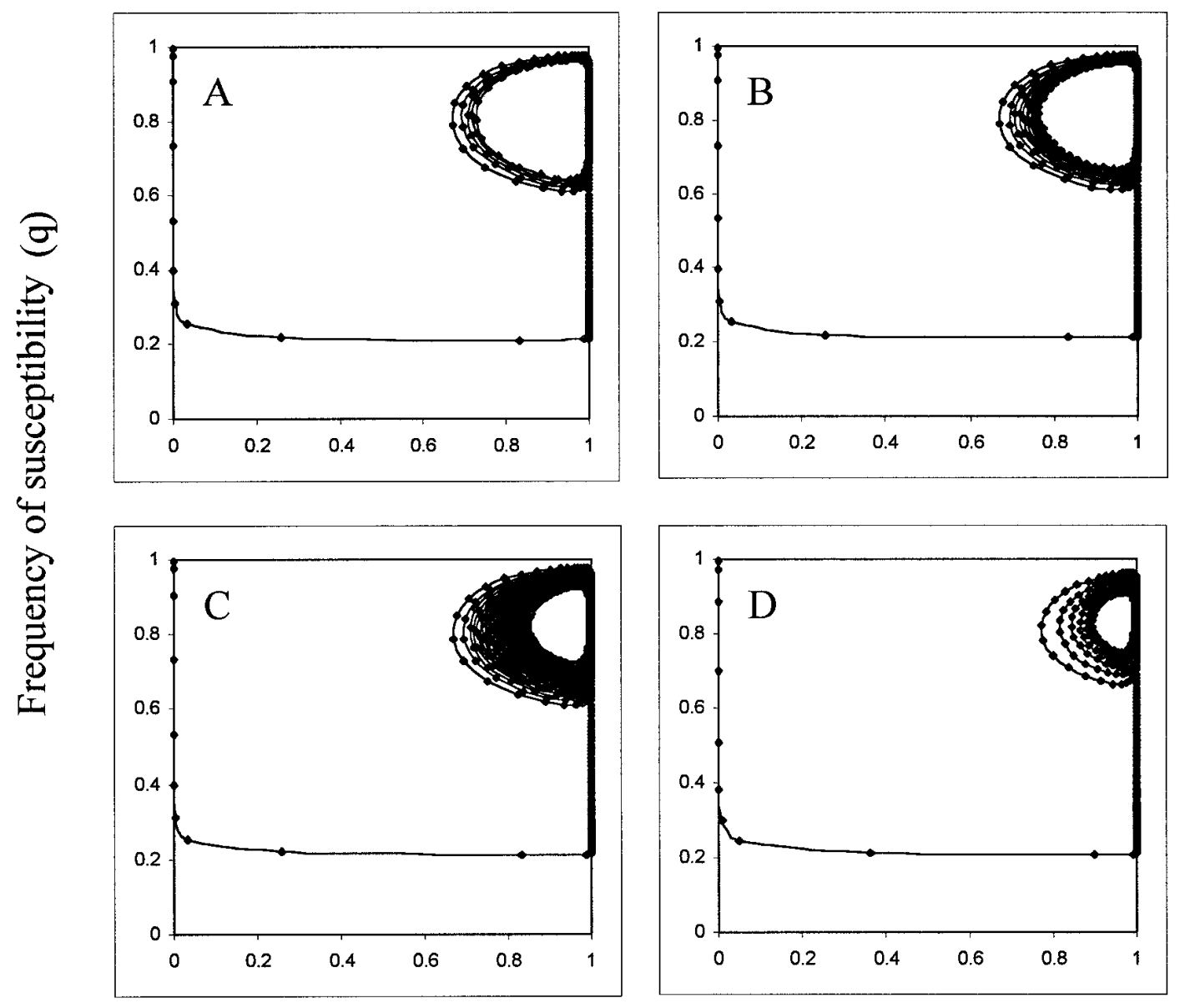

\section{Frequency of virulence (n)}

Fig. 1. Phase planes from simulations on the modified Leonard model comparing the effects of human perturbations $(\lambda)$ and natural perturbations (mutation $\mu$ ) on the stability of host-parasite interaction. The system is described by equation set 5 (described in text) at a set of parameters, $k=0.3, a=0, t=1, c=0.03$, and $s=0.8$. The frequency of susceptibility in the host population $q(q=1-p)$ is plotted against $n$, the frequency of the virulence in the parasite population. A, $\mu=$ $10^{-6}$ and $\lambda=0,500$ generations; $\mathbf{B}, \mu=10^{-6}$ and $\lambda=0,1,000$ generations; $\mathbf{C}, \mu=10^{-6}$ and $\lambda=0,5,000$ generations; and $\mathbf{D}, \mu=10^{-6}$ and $\lambda=0.001,500$ generations. The initial frequencies of $\left(n_{0}, q_{0}\right)$ are $(0.001,0.999)$. 
larger than the absolute values of $\mu\left[1-2 f\left(p_{i}, n_{i+1}\right)\right]$ and $\mu\left[1-2 g\left(p_{i}\right.\right.$, $\left.n_{i}\right)$ ]. Phase planes on the simulations of equation set 5 with parameter values $k=0.3, a=0, t=1, c=0.03$, and $s=0.8$ were derived (Fig. 1). Four different values for $\mu$ and $\lambda$ were used: (i) $\mu=$ $10^{-6}$ and $\lambda=0,500$ generations (Fig. 1A), which is the duplication of Figure 1C of Kirby and Burdon's work (8); (ii) $\mu=10^{-6}$ and $\lambda=0$, 1,000 generations (Fig. 1B); (iii) $\mu=10^{-6}$ and $\lambda=0,5,000$ generations (Fig. 1C); and (iv) $\mu=10^{-6}$ and $\lambda=0.001,500$ generations (Fig. 1D). The initial frequencies of $\left(n_{0}, q_{0}\right)$ are $(0.001,0.999)$. From the figures, we can see that in the absence of human perturbations the mutation effect could increase the rate of convergence toward the internal EP (Fig. 1A to C). However, if human perturbation, $\lambda$, is introduced, the convergence rate would increase significantly (Fig. 1D).

Effects of overuse of resistant genotypes. Equation set 6 was simulated by setting the parameter values as follows: $k=0.3, a=0$, $t=1, c=0$, and $s=0.2$, which is modified from the parameter set used in Figure 1. Modification was made by letting $c=0$ and $s=$ 0.2 , in which $c=0$ means no difference in yield between the two host genotypes in the absence of disease, and $s=0.2$ means the disease severity is low. There are four different suppositions about $\lambda_{i}$. The first two are as follows: (i) $\lambda_{1}=0.005, \lambda_{2}=0.010, \lambda_{3}=$ $0.015, \ldots, \lambda_{i+1}=0.005 \times(i+1)$, for $i=0$ to 14 (Fig. 2A); and (ii) $\lambda_{1}=0.01, \lambda_{2}=0.02, \lambda_{3}=0.03, \ldots, \lambda_{i+1}=0.01 \times(i+1)$, for $i=0$ to
10 (Fig. 2B). In both suppositions, the level of perturbation $\lambda_{i}$ is an increasing linear function of generation $i$. In the third supposition, the level of perturbation $\lambda_{i}$ is a geometrically increasing function of generation $i$ : (iii) $\lambda_{1}=0.01, \lambda_{2}=0.02, \lambda_{3}=0.04, \ldots$, $\lambda_{i+1}=0.01 \times 2^{i}$, for $i=0$ to 6 (Fig. 2C). The fourth supposition corresponds to another kind of human perturbation, the densitydependent perturbation, of which the mathematical description is different from equation set 6 and is written as follows.

$$
\begin{gathered}
n^{*}{ }_{i+1}=n_{n+1}=g\left(p_{i}, n_{i}\right) \\
p^{*}{ }_{i+1}=f\left(p_{i}, n_{i+1}\right) \\
p_{i+1}=f\left(p_{i}, n_{i+1}\right)+\lambda_{i+1}
\end{gathered}
$$

with $\lambda_{i+1}=\lambda f\left(p_{i}, n_{i+1}\right)$ and $\lambda=0.1$, for $i=0$ to 10 (Fig. 2D). In the simulation for the four suppositions, because of the drastic increase in the value of $\lambda_{i}$, there are quite large differences between the frequencies of $\left(n_{i}, p_{i}\right)$ and $\left(n^{*}, p^{*}{ }_{i}\right)$, that is, the initial frequencies of the $(i+1)$ th generation and the final frequencies of the $i$ th generation. In Figure 2, the simulation results on the frequencies of $\left(n{ }_{i}, p^{*}\right)$ are presented.
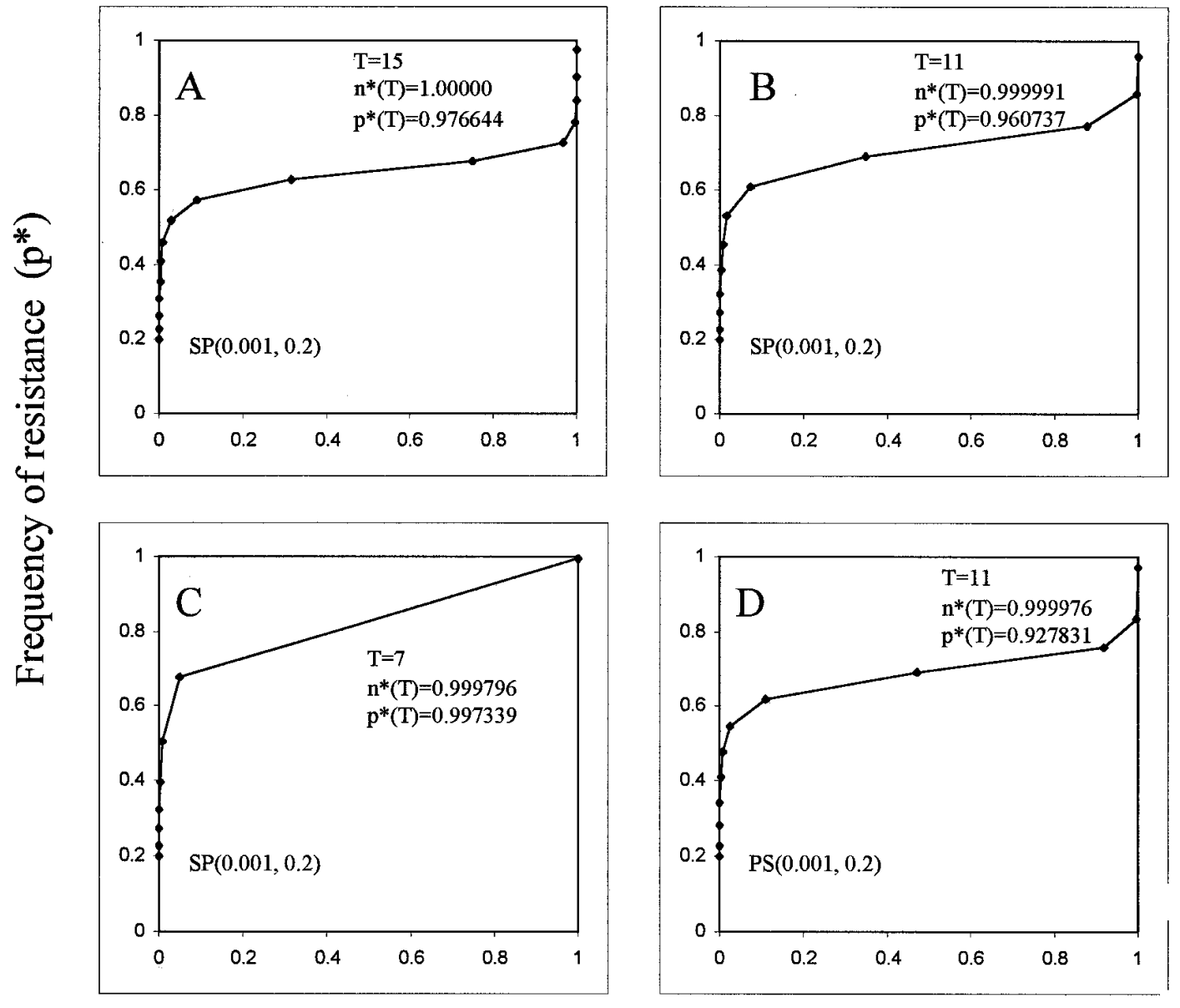

\section{Frequency of virulence $\left(\mathrm{n}^{*}\right)$}

Fig. 2. A to C, Phase planes of $\left(n_{i}^{*}, p_{i}^{*}\right)$, frequencies of virulence in the parasite population and resistance in the host population at the end of the growing season $i$, from simulations on the overuse of a resistant genotype that can be overcome by a virulent parasite. The system is described by equation set $6, n_{i+1}=n^{*}{ }_{i+1}=g\left(p_{i}, n_{i}\right)$, $p_{i+1}=f\left(p_{i}, n_{i+1}\right)+\lambda_{i+1}$, and $p_{i+1}=f\left(p_{i}, n_{i+1}\right)$, for $i=0,1,2, \ldots$, at parameters values of $k=0.3, a=0, t=1, c=0$, and $s=0.2 . T$ is the number of simulations. A, $\lambda_{i+1}=$ $0.005 \times(i+1)$, for $i=0$ to 14 and $T=15 ; \mathbf{B}, \lambda_{i+1}=0.01 \times(i+1)$, for $i=0$ to 10 and $T=11$; and $\mathbf{C}, \lambda_{i+1}=0.01 \times 2^{i}$, for $i=0$ to 6 and $T=7$. D, Phase plane from simulations of equation set $7, n_{i+1}=n{ }^{*}{ }_{i+1}=g\left(p_{i}, n_{i}\right), p_{i+1}=(\lambda+0.1) \times f\left(p_{i}, n_{i+1}\right)$, and $p^{*_{i+1}}=f\left(p_{i}, n_{i+1}\right)$, for $i=0$ to 10 and $T=11$. SP is the initial frequencies of the simulation. $p^{*}(T)$ and $n^{*}(T)$ are the frequencies of the resistant host and the virulent parasite, respectively, at the end of generation $T$. 
The simulations under suppositions $i$ and ii show that the greater the increase in generational addition of resistant genotypes (larger $\lambda_{i}$ ), the faster the frequencies of resistance and virulence move toward $p^{*}{ }_{i}=1$ and $n{ }_{i} \approx 1$ (the quicker the resistant genotype is "busted"). When the increment of the resistant genotype in each generation is linear with an increment value of 0.005 (supposition i) and when the increment is 0.01 (supposition ii), it takes 15 generations and 11 generations, respectively, for the host-parasite system to reach $p^{*}{ }_{i}=1$ and $n_{i} \approx 1$ (Fig. $2 \mathrm{~A}$ and $\mathrm{B}$ ). In the third supposition, with geometric increase in $\lambda_{i}, \lambda_{i}=\left(0.01 \times 2^{i}\right)$, for $i=0$, $1,2, \ldots$, within seven generations the host-parasite system will then reach $n^{*}{ }_{i} \approx 1$ and $p^{*}{ }_{i}=1$ (Fig. 2C). The simulation with equation set 7 (density-dependent perturbation) shows that the system will reach $n^{*}{ }_{i} \approx 1$ and $p{ }_{i}=1$ at 11 generations (Fig. 2D) when $\lambda=0.1$. However, smaller values of $\lambda$ in equation set 7 will lead the system to converge to the internal EP. For example, if $\lambda=$ 0.02 , or 0.03 in equation 7 , the frequencies of $\left(n *_{i}, p^{*}\right)$ converge to the internal EP, not to $(1,1)$, which implies that limited use rather than overuse can sustain resistance of a genotype. Theoretically, there is a threshold value of $\lambda_{0}$ for equation set 7 . When $\lambda<\lambda_{0}$, the system will converge to its internal EP. However, it is difficult to derive the value of $\lambda_{0}$.

To evaluate the effects of parameter $s$, we simulated equation sets 6 and 7 under the above four suppositions by changing the model parameter $s=0.2$ to $s=0.1$ and 0.3. When $s=0.1$, it took 16, 12, 7 , and 14 generations for the frequencies of $\left(n^{*}, p_{i}^{*}\right)$ to approach $(1,1)$, and when $s=0.3$, it took $14,10,6$, and 10 generations for the frequencies of $\left(n{ }_{i}, p^{*}\right)$ to approach $(1,1)$. It takes longer for the frequencies of $\left(n^{*}, p_{i}{ }^{*}\right)$ to approach $(1,1)$ under lower disease severity $(s=0.1)$. The slope of the graphs of $\left(n^{*}, p_{i}^{*}\right)$ approaching $(1,1)$ under $s=0.1$ or 0.3 are similar to $s=0.2$ (data not shown).

A common feature of the four suppositions is that the frequencies of virulence in the parasite population increased slowly in the first three to four generations and then increased rapidly (from 0.01 to $>0.5$ ) within a few generations (Fig. 2). This frequency change is consistent with the theoretical descriptions of the "boom and bust" phenomena $(1,7,13,14,20,21)$.

\section{DISCUSSION}

In using Leonard's model, it is assumed that the pathogen genotypes are randomly distributed among resistant and susceptible host genotypes. For some airborne pathogens, the random association might be closely approximated within a larger agroecosystem. For example, in wheat fields in China, each farmer has a small piece of land, and farm lands are connected. Some fields are planted with resistant genotypes, some are planted with susceptible genotypes, and some are planted with both. Currents carry pathogen spores into the air and they are then deposited onto the fields. The mixing of host and pathogen genotypes is approximately (or close to) random (22).

To describe a "boom and bust" epidemic cycle in the real world with the modified Leonard model, further definition of the parameters may be necessary according to the characteristics of the pathogen and the host involved, and some parameters need to be evaluated in each generation. For example, parameter $s$, disease severity, partly depends on weather conditions. If in a growing season the climate is ideal for infection, disease severity could be much higher than in other seasons.

Leonard's model was originally developed to describe coevolution of host-parasite interactions in a natural system, in which frequencies of the resistance (or the susceptibility) of the host and the virulence (or the avirulence) of the parasite population at the end of one generation were used as the initial frequencies for the next generation. When certain perturbations are taken into consideration, we need to modify Leonard's model to describe the more complex coevolution process. The extension is reasonable because, in Leonard's model (equation set 1), the interaction between the frequencies of $\left(n_{i+1}, p_{i+1}\right)$ and $\left(n_{i}, p_{i}\right)$ is actually determined by the parameters of the model, but not the sources of $\left(n_{i}, p_{i}\right)$. Suppose, for instance, the initial frequencies are $\left(n_{0}, p_{0}\right)$, equation set 1 can be used to calculate the frequencies of $\left(n_{1}, p_{1}\right)$ regardless of whether the initial frequencies of $\left(n_{0}, p_{0}\right)$ existed in the system already or are experimentally determined by human beings. For another example, suppose at the end of the $i$ th generation $n_{i}=0.3, p_{i}=0.4$, then in the next generation, either the initial frequencies are $(0.3,0.4)$ or $(0.3$, $0.4+\lambda)(\lambda$ represents a human perturbation), the frequencies at the end of the generation can be calculated by the formula $n_{i+1}=g(0.4$, $0.3)$ and $p_{i+1}=f\left(0.4, n_{i+1}\right)$ if the initial frequencies are $(0.3,0.4)$, or $n_{i+1}=g(0.4+\lambda, 0.3)$ and $p_{i+1}=f\left(0.4+\lambda, n_{i+1}\right)$ if the initial frequencies are $(0.3,0.4+\lambda)$.

Many theoretical models are developed to describe the coevolution process of host-parasite interactions $(6,18)$. However, it is difficult to validate these models because in real world systems these interactions are always perturbed by some complex factors, which produce deviations from behaviors described by the theoretical models $(2,3,16,19)$. It is very difficult to create environments free of perturbations for long-term experiments. However, models can be tested by other ways, based on common knowledge of biology and experiences of experts. It is generally recognized that the evolution of a natural ecosystem is a very slow process, but the process can be sped up and the direction of evolution could be changed when the system is subjected to human perturbations. The results of comparing simulations of human perturbations and natural perturbations are consistent with this well-known belief. From the simulation of the overuse of pure line resistant genotypes, we can see that the model has the potential to predict the "boom and bust" epidemic cycles (Fig. 2A to D). Because the simulation results on equation set 6 produced the typical "boom and bust" patterns observed in many pathosystems (7), the properties of Leonard's model revealed in this paper may describe some properties of pathosystems in the real world.

Analyzing data of real "boom and bust" events in light of Leonard's model would be interesting. Further development of Leonard's model may be needed so that the model can describe population dynamics under different ecological scales. An unstated assumption on the original Leonard model (equation set 1) and the modified Leonard model (equation set 6) is that the host and the parasite interact in a closed system, in which the resistant/susceptible host genotypes and the virulent/avirulent parasite genotypes are randomly distributed, and there is no migration of a pathogen into the system. In reality, the condition on pathogen movement could be satisfied in microcosm experiments or can be approximately satisfied in a large epidemic system that the pathogen movement is limited within. However, the parameter estimation of the models might be different under different ecological scales. Another difficulty in improving Leonard's model to describe or predict real "boom and bust" epidemic cycles would be the lack of field data. Quality data of long-term host-parasite interaction are scarce for model modification and validation. For example, wheat stripe rust (Puccinia striiformis) was a severe disease in China in the period of the 1960s to 1980 s, and use of resistant cultivars that had a few major genes was the main control strategy. The overuse of resistant cultivars of a few major genes resulted in "boom and bust" epidemic cycles during that period (12). Though the historic events in China have been documented $(12,22,23)$, data are not detailed enough to test the modified Leonard model.

\section{ACKNOWLEDGMENTS}

Journal Paper No. J-18337 of the Iowa Agricultural and Home Economics Experiment Station. We thank K. Leonard for valuable suggestions and S. Sanogo for critical presubmission review.

\section{LITERATURE CITED}

1. Agrios, G. N. 1988. Plant Pathology. 3rd ed. Academic Press, New York.

2. Bremen, A. A., and Millstone, J. A. 1989. Are ecological systems cha- 
otic-And if not, why not? Trends Ecol. Evol. 4:26-28.

3. Coven, J. E. 1995. Unexpected dominance of high frequencies in chaotic nonlinear population models. Nature 378:610-612.

4. Frank, S. A. 1993. Coevolutionary genetics of plants and pathogens. Evol. Ecol. 7:45-75.

5. Hamilton, W. D. 1993. Haploid dynamic polymorphism in a host with matching parasites: Effects of mutation/subdivision, linkage and patterns of selection. J. Hered. 84:328-338.

6. Hochberg, M. E., Hassell, M. P., and May, R. M. 1990. The dynamics of host-parasitoid-pathogen interactions. Am. Nat. 135:74-79.

7. Jones, D. G. 1987. Plant Pathology, Principles and Practices. PrenticeHall, Englewood Cliffs, NJ.

8. Kirby, G. C., and Burdon, J. J. 1997. Effects of mutation and random drift on Leonard's gene-for-gene coevolution model. Phytopathology 87:488-493.

9. Leonard, K. J. 1977. Selection pressures and plant pathogens. Ann. N.Y. Acad. Sci. 287:207-222.

10. Leonard, K. J. 1994. Stability of equilibria in a gene-for-gene coevolution model of host-parasite interactions. Phytopathology 84:70-77.

11. Leonard, K. J. 1997. Modelling gene frequency dynamics. Pages 221230 in: The Gene-for-Gene Relationship in Plant-Parasite Interactions. I. R. Crute and E. B. Holub, eds. CAB International, Wallingford, United Kingdom.

12. Li, Z. Q. 1989. The Wheat Stripe Rust and Its Control. Shanghai Science and Technique Publishing Inc., Shanghai, China.

13. Priestley, R. H. 1978. Detection of increased virulence in populations of yellow rust. Pages 63-70 in: Plant Disease Epidemiology. P. R. Scott and A. Bainbridge, eds. Blackwell Scientific, Oxford.

14. Robinson, R. A. 1976. Plant Pathosystems. Springer-Verlag, New York.

15. Robinson, R. A. 1987. Host Management in Crop Pathosystems. CollierMacmillan, New York.

16. Stone, L. 1992. Coloured noise or low-dimensional chaos? Proc. R. Soc. B 250:77-81.

17. Sun, P., and Yang, X. B. 1998. Stability of a gene-for-gene coevolution system under constant perturbations. Phytopathology 88:592-597.

18. Thompson, J. N., and Burdon, J. J. 1992. Gene-for-gene coevolution between plants and parasites. Nature 360:121-125.

19. Turchin, P., and Taylor, A. D. 1992. Complex dynamics in ecological time series. Ecology 73:289-305.

20. Ullstrup, A. J. 1972. The impacts of the southern corn leaf blight epidemics of 1970-1971. Annu. Rev. Phytopathol. 10:37-50.

21. Zadoks, J. C., and Schein, R. D. 1979. Epidemiology and Plant Disease Management. Oxford University Press, New York.

22. Zeng, S. M. 1991. PANCRIN, a prototype model of the pandemic cultivarrace interaction of yellow rust on wheat in China. Plant Pathol. 40:287-295.

23. Zeng, S. M., and Yang, Y. 1980. Plant Disease Epidemiology. China Agricultural Publishing House, Beijing. 